\title{
GROUP ACTIONS ON THE COMPLEX PROJECTIVE PLANE
}

\author{
DARIUSZ M. WILCZYŃSKI
}

\begin{abstract}
Let $G$ be a finite or compact Lie group. It is shown that $G$ acts on the complex projective plane (resp. on the Chern manifold) if and only if $G$ is isomorphic to a subgroup (resp. a pseudofree subgroup) of $P U(3)$. All actions considered are effective, locally smooth, and trivial on homology.
\end{abstract}

0. Introduction. In this paper we consider homologically trivial, locally smooth actions of finite and compact Lie groups on topological 4-manifolds having the same homology as $\mathbf{C} P^{2}$, the complex projective plane. Our main result states that all such actions are locally complex linear and that the acting groups can also act linearly on $\mathrm{C} P^{2}$. In particular, we obtain the classification of groups acting on both manifolds homotopy equivalent to $\mathbf{C} P^{2}: G$ acts on the complex projective plane (resp. on the Chern manifold) if and only if $G$ is isomorphic to a subgroup (resp. a pseudofree subgroup) of $P U(3)$.

The paper is organized as follows. In $\S 1$ a precise statement of the main result is given. $\$ 2$ examines tangent representations of some cyclic groups; the main tool being the $G$-signature theorem. The relevant number theoretic computations are carried out in Appendix 1. $\S \S 3$ and 4 discuss abelian and nonabelian groups in terms of the numbers of fixed points (Euler characteristic of the fixed point set). $\$ 5$ proves that all tangent representations are complex. $\S \S 6-9$ discuss several classes of groups acting without fixed points. The terminology that we use to distinguish among different classes of fixed point free groups is adopted from the linear case. $\$ 10$ contains the proof of our main theorem and, finally, $\S 11$ is devoted to group actions on the Chern manifold. For completeness, a list of fixed point free linear groups is included in Appendix 2.

Acknowledgment. I would like to thank Professor John Ewing for suggesting this problem to me and also for his support and encouragement.

I have been informed that I. Hambleton and R. Lee have independently obtained results similar to those presented here.

1. Statement of the main result. Let $M$ be a closed topological manifold having the same integral homology as $\mathbf{C} P^{2}$. It follows that $M$ is an orientable 4-manifold with a perfect fundamental group. We assume $M$ is oriented so that $\left\langle\alpha^{2},[M]\right\rangle=1$, where $\alpha \in H^{2}(M)$ is a generator. If $\pi_{1}(M)=0, M$ is homotopy equivalent to $\mathbf{C} P^{2}$.

Received by the editors October 2, 1986.

1980 Mathematics Subject Classification (1985 Revision). Primary 57S25.

"1987 American Mathematical Socicty $0(0) 2-9947 / 87 \$ 1.00+\$ .25$ per page 
If, in addition, $M$ is smooth (or PL), then by Freedman [F1] it is homeomorphic to $\mathrm{C} P^{2}$.

It is a consequence of the cohomology ring structure that every homeomorphism $M \rightarrow M$ preserves orientation and, by the Lefschetz fixed point theorem, has a fixed point. In particular, there are no free group actions on $M$.

We are interested in the actions of finite or more generally of compact Lie groups on $M$ that induce a trivial action on homology. If the action is effective, the acting group can be identified with a subgroup of $\mathscr{G}^{+}(M)$, the group of all homeomorphisms acting trivially on homology, endowed with the compact-open topology. We write $G<\mathscr{G}^{+}(M)$, if $G \subseteq \mathscr{G}^{+}(M)$ is a compact Lie group and the $G$-action on $M$ is locally smooth.

Given $G<\mathscr{G}^{+}(M)$ and a fixed point $x \in M^{(i}$, each group element $g$ acts, by means of its differential $d g$, on the tangent space to $M$ at $x$. Since $G$ acts effectively on $M$, the resulting linear representation is faithful. It should be noted that this tangent representation of $G$ is uniquely defined (up to linear equivalence) even when the action is only locally smooth. Indeed, in the case of a locally smooth action the tangent representation is determined up to topological equivalence (cf. [S, §3]) but topological equivalence in dimension 4 coincides with linear equivalence by [CS]. In particular, this will allow us to include in the discussion locally smooth actions on nonsmoothable 4-manifolds.

Our main result is the following.

THEOREM 10.1. Every group $G<\mathscr{G}^{+}(M)$ is isomorphic to a subgroup of $P U(3)$ and the action of $G$ is locally complex linear.

The phrase a locally complex linear action refers to the following property: given a subgroup $H \subseteq G$ and a fixed point $x \in M^{H}, H$ preserves a complex structure on the tangent space at $x$, that is, the tangent $H$-representation at $x$ is a 2-dimensional complex representation.

2. Tangent representations of cyclic groups. In this section $G<\mathscr{G}^{+}(M)$ denotes a cyclic group generated by an element $g$ of finite order $n$. By Smith theory, each connected component of the fixed point set $M^{G}$ is a homology $\mathbf{C} P^{k}$ with $k=0$ or 1. Since $\chi\left(M^{G}\right)=L(g)=3, M^{(i}$ consists of three isolated points or consists of a single point and a 2-sphere. In the latter case $g$ is called a homology. In particular, every involution is a homology. This follows from [B, Theorem VII.3.1]. On the other hand, if $G$ contains no homology, except possibly an involution, then we call $g$ a cohomology.

It will be important in our analysis of subgroups of $\mathscr{G}^{+}(M)$ to be able to distinguish between homologies and cohomologies by means of a single tangent representation. This is, roughly speaking, the content of Propositions 2.3 and 2.4.

We start with the following.

Proposition 2.1. If $g$ is a homology, the 2-sphere fixed by $g$ represents a generator of $\mathrm{H}_{2}(\mathrm{M})$. 
Proof. Suppose the sphere represents $k \mu$, where $\mu \in H_{2}(M)$ is a generator. By passing to a power of $g$, if necessary, we can assume that $n$ is a prime number. By [B, Theorem VII.3.1], $k \neq 0$.

Let $\alpha, \beta$ be the rotation angles of $g$ at the isolated fixed point and let $\theta$ be the angle of rotation in the normal direction along the fixed 2-sphere. By the AtiyahSinger $G$-signature theorem (see [AS, p. 585]),

$$
k^{2} \cdot \operatorname{cosec}^{2}(\theta / 2)-\cot (\alpha / 2) \cot (\beta / 2)=1 .
$$

Since $\omega=e^{i \theta}$ is a primitive $n$th root of unity, there are integers $a, b$ such that $e^{i \alpha}=\omega^{a}, e^{i \beta}=\omega^{h}$. The above condition can be rewritten as'

$$
k^{2} \cdot \frac{-4 \omega}{(\omega-1)^{2}}+\left(\frac{\omega^{a}+1}{\omega^{a}-1}\right)\left(\frac{\omega^{b}+1}{\omega^{b}-1}\right)=1 \text {. }
$$

For $n=2$, this clearly implies $k^{2}=1$.

Suppose now $n>2$. Multiplying both sides of $(*)$ by $(\omega-1)^{2}$, we obtain

$$
\left(\omega^{a}+1\right)\left(\omega^{b}+1\right)\left(\frac{\omega-1}{\omega^{a}-1}\right)\left(\frac{\omega-1}{\omega^{b}-1}\right)=\omega^{2}+\left(4 k^{2}-2\right) \omega+1 .
$$

The left-hand side now becomes a unit in the ring of algebraic integers $\mathbf{Z}[\omega]$. On the other hand, $\left|z^{2}+\left(4 k^{2}-2\right) z+1\right|>1$ for any $|z|=1$ and $k^{2}>1$. Consequently, the norm of $\omega^{2}+\left(4 k^{2}-2\right) \omega+1$ in $\mathbf{Z}[\omega]$ is not \pm 1 unless $k^{2}=1$.

Remark. Although stated in [AS] only in the differentiable category, the $G$ signature theorem applies to locally smooth actions on topological 4-manifolds as well. This follows from [F2] and [W, p. 188] (see also [D, Theorem 1.1]). Wall's argument assumed that the action was semifree but one can use bordism groups of adjacent families of subgroups of $G$ and exact sequences thereof (see $[\mathbf{C F}]$ ) to extend this argument to general actions.

Definition 2.2. Given $x \in M^{G}$ and rotation angles $\alpha, \beta$ of $g$ at $x$ ( $g$ is not necessarily a homology here), we say that $g$ has a single (complex) eigenvalue at $x$ if $\alpha-\beta=0$ modulo $2 \pi$. Similarly, we say that $g$ has a pair of conjugate eigenvalues at $x$ if $\alpha+\beta=0$ modulo $2 \pi$.

Since the tangent representation at each point is not a priori complex but only an oriented real representation, there is some ambiguity in the choice of the angles $\alpha, \beta$. For instance, if $x$ is an isolated fixed point then one may choose $(-\alpha,-\beta)$ or $(\beta, \alpha)$ instead of $(\alpha, \beta)$. However, $\alpha \pm \beta$ does or does not equal 0 independently of these choices. The same remark applies to nonisolated fixed points.

Proposition 2.3. Every homology $g$ has a single eigenvalue at the isolated fixed point. This eigenvalue is a complex conjugate of that in the normal direction along the 2-sphere fixed by $g$.

Proof. As noted before, the $G$-signature theorem leads to the condition

$$
=\frac{4 \omega}{(\omega-1)^{2}}+\left(\frac{\omega^{a}+1}{\omega^{a}-1}\right)\left(\frac{\omega^{b}+1}{\omega^{b}-1}\right)=1
$$


or

$$
\left(\frac{\omega^{a}+1}{\omega^{a}-1}\right)\left(\frac{\omega^{b}+1}{\omega^{b}-1}\right)=\left(\frac{\omega+1}{\omega-1}\right)^{2},
$$

where $\omega$ is a primitive $n$th root of unity. Hence, by Proposition A.1 (see Appendix 1), $\omega^{a}=\omega^{h}=\omega^{ \pm 1}$.

PROPOSITION 2.4. If $g$ is a cohomology and two of its tangent representations are equivalent, then $g$ has a pair of conjugate eigenvalues at the third fixed point.

Proof. This is an immediate consequence of the $G$-signature theorem and Proposition A.2.

It should be noted that the question of which local fixed point data can be realized for periodic maps on complex projective spaces in the presence of a codimension 2 fixed point set component, has been also discussed in some low dimensional cases by Dovermann. In [D], he proved a version of Proposition 2.3 for prime orders.

Finally, let us note that Propositions 2.3 and 2.4 hold for $G=S O(2)$ as well. This follows from the fact that every character is a continuous function.

3. Abelian groups. By analogy with the linear case, we introduce the following terminology.

Definition 3.1. A subgroup $G<\mathscr{G}^{+}(M)$ having no fixed points on $M$ is called irreducible; otherwise $G$ is called reducible.

Proposition 3.2. Suppose $G<\mathscr{G}^{+}(M)$ is an irreducible abelian group. Then $G$ is an elementary abelian group of order 9 that contains no homology.

Proof. The last statement is clear. For if there were a homology $g \in G$, then $G$, acting on $M^{g}$, would have to fix the isolated point. In particular, this forces $G$ to be a finite group of odd order.

Fix $g \in G, g \neq 1$. The action of $G$ on $M^{g}$ defines a homomorphism $\varphi: G \rightarrow S_{3}$, where $S_{3}$ is the symmetric group on three letters. Let $H=\operatorname{Ker} \varphi$. The irreducibility hypothesis implies that $G$ is a noncyclic group such that $G / H=C_{3}$, a cyclic group of order 3. Furthermore, each element $u \in G-H$ has order 3 or else $G$ would fix every point fixed by $u^{3}$. This implies that $G=H \times C_{3}$ and $H=\left(C_{3}\right)^{k}$ for some $k \geqslant 1$. Since $H$ contains no homology, $M^{h}=M^{H}$ for each $h \in H$ and therefore each tangent representation of $H$ is free off the origin 0 . Thus $H$ acts freely on a 3-dimensional sphere and hence is a cyclic group (see [B, Theorem III.8.2]).

As a corollary, we obtain a bound for the $p$-rank of $\mathscr{G}^{+}(M)(=$ the maximal rank of all elementary abelian $p$-subgroups).

COROllaRy 3.3. Let $p$ be a prime number. Then p-rank $G \leqslant 2$ for every group $G<\mathscr{G}^{+}(M)$.

Proof. Suppose $K=\left(C_{p}\right)^{k} \subseteq G$. We want to show $k \leqslant 2$. If $K$ is irreducible, then $p=3$ and $k=2$ by Proposition 3.2. Otherwise, $K$ has a fixed point, and hence a faithful oriented 4-dimensional representation. Consequently, $k \leqslant 2$ for $p>2$. For $p=2$, we use the hypothesis that the action is trivial on homology, which implies that every subgroup of $K$ acts in an orientation preserving way on every 
2-sphere fixed by an element of $K$. It follows that in the tangent representation of $K$ every subgroup fixes a subspace of even dimension. Therefore $k \leqslant 2$ in this case as well.

We are going to show now that, unlike the irreducible group in Proposition 3.2, a noncyclic abelian reducible group $H<\mathscr{G}^{+}(M)$ always contains some homologies.

Proposition 3.4. Assume $H<\mathscr{G}^{+}(M)$ is a reducible group isomorphic to $C_{n} \times C_{n}$ for some $n$. Then $H$ is generated by two homologies $x$ and $y$ such that $z=x y$ is also $a$ homology. The isolated fixed points of $x, y, z$, are distinct and fixed by $H$. Furthermore, $H$ has no other fixed points and consequently any homology $h \in H$ is a power of $x, y$ or $z$.

Proof. Let $V$ be a tangent representation of $H$. It splits into a direct sum $V_{1} \oplus V_{2}$ of two oriented 2-dimensional representations. Let $\chi_{i}: H \rightarrow S^{1}$ be the character of $V_{i}$ and let $K_{i}=\operatorname{ker} \chi_{i}(i=1,2)$.

Choose $x, y \in H$ so that $K_{1}=\langle x\rangle, K_{2}=\langle y\rangle$ and $\chi_{2}(x)=\chi_{1}(y)$. It follows that $x, y$ are homologies that generate $H$.

Consider the action of $H$ on $M^{x}$. Of course, $H$ preserves the isolated point in $M^{x}$. Also, since $H / K_{1}$ is a cyclic group, the fixed point set of $H$ on the 2-sphere in $M^{x}$ is either the whole sphere or consists of two isolated points. The group $H$ cannot fix a 2-sphere, however, because the normal representation along the sphere would be its faithful oriented representation of dimension 2 and this would force $H$ to be a cyclic group. Hence $H$ has precisely three fixed points.

Two of these points lie also on the 2-sphere fixed by $y$, but one of them must be the isolated fixed point of $x$ or else the fixed 2-spheres would intersect at exactly two points. Since both spheres represent a generator of $H_{2}(M)$ and consequently have intersection number \pm 1 , this would be a contradiction. In particular, the isolated fixed points of $x$ and $y$ do not coincide.

According to Proposition 2.3, $x$ has a single eigenvalue at the isolated fixed point. Since this eigenvalue is a complex conjugate of $\chi_{2}(x)=\chi_{1}(y), z=x y$ must have an eigenvalue 1 at this point, and hence $z$ is a homology. The same argument as before shows that the isolated fixed points of $x, y, z$ are distinct and therefore $H$ has no other fixed points.

Finally, suppose $h \in H$ is a homology. Its isolated fixed point is fixed by $H$, hence it coincides with the isolated fixed point of $x, y$ or $z$. Assume, without loss of generality, that $h$ and $x$ have the same isolated fixed point. Since the 2-spheres fixed by $h$ and $x$ cannot intersect at two points, they must coincide. It follows that $\langle h, x\rangle$ is a cyclic group generated by a homology. Since $x$ has a maximal order in $H$, $\langle h, x\rangle$ is generated by $x$.

Elements $x, y \in H$ with the properties as above will later be referred to as coordinate homologies.

Proposition 3.5. Let $G<\mathscr{G}^{+}(M)$ be an abelian reducible group. If p-rank $G>1$ for some $p$, then $G$ has precisely three fixed points.

Proof. By assumption, there exists a subgroup $H \subseteq G$ isomorphic to $C_{p} \times C_{p}$. Then $M^{G} \subseteq M^{H}$. The opposite inclusion follows from Proposition 3.4. 
4. Nonabelian reducible groups. In this section we study nonabelian reducible subgroups of $\mathscr{G}^{+}(M)$. These turn out to be groups having a single fixed point. This, together with the results of the previous section, leads to some useful information about normalizers of abelian reducible subgroups.

Proposition 4.1. Let $G<\mathscr{G}^{+}(M)$. Suppose $H \subseteq G$ is a normal closed subgroup generated by a single homology $h \in G$ and $K \subseteq G$ is a subgroup containing $H$. Then $K$ is a nonabelian group if and only if it has precisely one fixed point.

Proof. $G$ operates on $M^{H}$ preserving the isolated point. If $K$ fixes more than one point, it has a fixed point lying on the 2-sphere in $M^{H}$. The tangent representation of $K$ at this point splits into a direct sum of two oriented representations, each of dimension 2, and therefore $K$ must be an abelian group. The converse follows from Proposition 3.5 .

Corollary 4.2. Under the hypothesis of Proposition 4.1, $G=Z_{G}(H)$, the centralizer of $H$ in $G$.

Proof. Every $g \in G$ operates on $M^{H}$ having at least two fixed points on the 2-sphere. Consequently, $K=\langle g, h\rangle$ is an abelian group and $g \in Z_{G}(H)$.

REMARK 4.3. If $h \in G$ is a central homology, then the isolated point of $M^{h}$ is necessarily fixed by all elements of $G$. We shall see later that the converse is also true (Proposition 5.6). This is immediate, however, in the case of an involution. Indeed, consider the tangent representation of $G$ at the isolated point of $M^{h}$. Then $h$ has a single eigenvalue -1 at this point, so it clearly commutes with all elements of $G$.

LEMMA 4.4. A dihedral group $D_{n}<\mathscr{G}^{+}(M)$ of order $2 n, n>2$ odd, fixes precisely one point.

Proof. $D_{n}=\left\langle x, u \mid x^{n}=u^{2}=1, u x u^{-1}=x^{-1}\right\rangle$. It follows from Corollary 4.2 that $x$ must be a cohomology. Hence $D_{n}$ fixes precisely one or three points. Suppose it fixes three. Since the same must hold for any dihedral subgroup of $D_{n}$, we can assume that $n$ is a prime number.

By Remark 4.3, the isolated point of $M^{r}$ is not fixed by $D_{n}$ for any involution $v \in D_{n}$. Moreover, since $\left\langle x^{k} u, x^{m} u\right\rangle=D_{n}$ for $x^{k} \neq x^{m}$, the 2-spheres fixed by $x^{k} u$ and $x^{m} u$ have precisely three points in common. Since $x^{k} u$ and $x^{m} u$ are conjugate in $D_{n}$, both spheres represent the same generator of $H_{2}(M)$, so their intersection number equals +1 . At one of the fixed points the intersection number must be -1 . Let $P$ be such a point for $u$ and $x^{2} u=x u x^{-1}$.

Let $V$ be the tangent plane at $P$ to the 2-sphere fixed by $u$. Then $x V$ is tangent to the 2-sphere fixed by $x^{2} u$. The generator of $H_{2}(M)$ determines the orientations of both spheres, hence the orientations of their tangent planes. Thus $x: V \rightarrow x V$ is orientation preserving, but the orientation on $V \oplus x V$ does not agree with the orientation of $M$ at $P$; let us denote this fact briefly by $[V, x V]=-1$. Since $x$ preserves the orientation of $M$, we also have $\left[x^{k} V, x^{k+1} V\right]=-1$ for each $k$. Hence, $\left[V, x^{h} V\right]=(-1)^{h}$ and, in particular, $\left[V, x^{n-1} V\right]=1$. But

$$
\left[V, x^{n-1} V\right]=\left[x V, x^{n} V\right]=[x V, V]=[V, x V]=-1
$$


by the choice of $P$. The contradiction proves that $D_{n}$ can have only one fixed point.

Before proving the main result of this section we recall from [Wo] that every closed subgroup of $S O(3)$ is conjugate to $S O(2), N(S O(2)), C_{n}, D_{n}$ or a polyhedral group and that there are three polyhedral groups (symmetries of the tetrahedron, octahedron, and icosahedron, respectively).

THEOREM 4.5. A nonabelian reducible group $G<\mathscr{G}^{+}(M)$ has precisely one fixed point.

Proof. Assume first that 2-rank $G=2$. Let $K \subseteq G$ be a subgroup isomorphic to $C_{2} \times C_{2}$. Then $K$ has three fixed points and at least one of them is fixed by $G$. But this point is also an isolated fixed point for one of the involutions in $K$. Therefore this involution must be central in $G$ (Remark 4.3), so Proposition 4.1 applies to $G$.

Assume that 2-rank $G \leqslant 1$. If $\operatorname{dim} G>0$, the identity component $G_{0}=S O(2)$ or $S U(2)$. Thus $G$ has a central involution, so again Proposition 4.1 applies. Hence we can assume that $G$ is a finite group. Let $G_{2}$ denote a 2-Sylow subgroup of $G$. If $G_{2}$ is nonabelian, it has a central involution, hence it has a single fixed point and so does $G$. Otherwise, $G_{2}$ is a cyclic group.

Since $G$ is reducible, it can be identified with a subgroup of $S O(4)$. There is a homomorphism

$$
p=\left(p_{1}, p_{2}\right): S O(4) \rightarrow S O(3) \times S O(3),
$$

whose kernel is $\{ \pm I\}$. Since $-I$ is a central element of $S O(4),-I \notin G$ or else Proposition 4.1 applies. Thus we can assume that $p$ embeds $G$ in $S O(3) \times S O(3)$. Since $G_{2}$ is cyclic, $p_{i}(G)$ is either a cyclic or dihedral group $(i=1,2)$. In the latter case $p_{i}(G)$ has a cyclic subgroup of odd order and index 2. Moreover, one of these two groups, say $p_{1}(G)$, must be dihedral as $G$ is nonabelian. Thus $p_{1}\left(G_{2}\right)=C_{2}$. If $G_{2} \cap \operatorname{Ker} p_{1} \neq(1)$, then $p_{2}(G)$ is a cyclic group since $G_{2}$ is assumed cyclic. Consequently, $G$ has a central involution in this case and Proposition 4.1 applies. Otherwise, $G_{2}$ has order 2 and elements of odd order form an abelian subgroup of index 2 in $G$. Then $G$ necessarily contains a nonabelian dihedral group which, according to Lemma 4.4 , has one fixed point.

Theorem 4.5 and Proposition 3.5 give the following.

Corollary 4.6. A reducible group $G<\mathscr{G}^{+}(M)$ is abelian if and only if $\chi\left(M^{G}\right)=3$.

Proposition 4.7. Suppose $G<\mathscr{G}^{+}(M)$ and $H \subseteq G$ is a reducible abelian subgroup. Then $N_{G}(H) / Z_{G}(H)$ is isomorphic to a subgroup of $D_{3}$. Furthermore, if $H$ is not generated by a single homology and $|H|>3$, then $Z_{G}(H)$ is a maximal abelian subgroup of $G$.

Proof. If $H$ is generated by a homology, then $N_{G}(H) / Z_{G}(H)=(1)$ by Corollary 4.2. Otherwise, $H$ has three fixed points. The action of $N_{G}(H)$ on $M^{H}$ defines a homomorphism of $N_{G}(H)$ into the symmetric group on three letters $S_{3}=D_{3}$. Let $K$ denote the kernel of this homomorphism. Since $K$ is abelian by Corollary 4.6 , 
$K \subseteq Z_{G}(H)$. Thus $N_{G}(H) / Z_{G}(H)$ is isomorphic to a subquotient of $D_{3}$, hence also to a subgroup of $D_{3}$. Moreover, if $|H|>3$, the subgroup $\langle g, H\rangle$, being abelian for any $g \in Z_{G}(H)$, must be contained in $K$ by Propositions 3.2 and 3.5. Hence $Z_{G}(H)=K$.

REMARK. If $H$ is generated by a homology, $Z_{G}(H)$ need not be abelian. Indeed, every binary dihedral or binary polyhedral subgroup of $P U(3)$ has a central involution but is a nonabelian group. The hypothesis that $H$ is a reducible group of order $>3$ cannot be relaxed either (see Appendix 2).

Proposition 4.8. If $G<\mathscr{G}^{+}(M)$ is a nonabelian (finite) $p$-group, then $p \leqslant 3$ and the center $Z(G)$ is a cyclic group. $Z(G)$ is generated by a homology for $p=2$ and by a nonhomology of order 3 for $p=3$.

Proof. By Smith theory, $\chi\left(M^{G}\right) \equiv 3 \bmod p$. If $p>3$, then $\chi\left(M^{G}\right)=3$ and $G$ would be abelian by Corollary 4.6. This shows that $p \leqslant 3$.

For $p=2, Z(G)$ has a fixed point. If $Z(G)$ were not generated by a homology, then it would contain either a nonhomology or a subgroup isomorphic to $C_{2} \times C_{2}$. In either case, $G$ would be abelian by Proposition 4.7.

Suppose now $p=3$. Being a nonabelian $p$-group $G$ has an abelian subgroup that properly contains $Z(G)$. Thus, by Proposition 3.2, $Z(G)$ is reducible. Moreover, $Z(G)$ contains no homology, for otherwise Proposition 4.1 would imply that $\chi\left(M^{G}\right)=1$. Hence, by Proposition $3.4, Z(G)$ is a cyclic group fixing three points. $G$ itself has no fixed points or else $\chi\left(M^{G}\right)=3$ and $G$ would be abelian. Thus $G$ contains an element $g$ which permutes the fixed points of $Z(G)$ as a 3-cycle. It follows that $\langle g, Z(G)\rangle$ is an irreducible abelian group. Hence, by Proposition 3.2, $\langle g, Z(G)\rangle=C_{3} \times C_{3}$, whence $|Z(G)|=3$.

5. Locally complex linear structure of group actions on $M$. To show that all group actions on $M$ are locally complex linear, we shall fix a reducible group $G<\mathscr{G}^{+}(M)$ and identify it with a subgroup of $S O(4)$ by means of the tangent representation at a selected fixed point. In $S O(4)$ we wish to find a copy of the unitary group $U(2)$ that contains $G$.

$S O(4)$ has two normal subgroups isomorphic to $S U(2)$. Using the terminology introduced in $\$ 1$, we can distinguish between these two subgroups by counting the (complex) eigenvalues of their elements. This can be seen more explicitly as follows. The multiplication of quaternions defines a map

$$
S^{3} \times S^{3} \times H \rightarrow H, \quad(a, b, q) \rightarrow a q b^{-1},
$$

which, in turn, gives rise to a Lie group homomorphism

$$
\psi: S^{3} \times S^{3} \rightarrow S O(4), \quad(a, b) \rightarrow\left(q \rightarrow a q b^{-1}\right) .
$$

The homomorphism $\psi$ is onto and $\operatorname{Ker} \psi=\{ \pm(1,1)\}$.

Proposition 5.1. Suppose $g \in S O(4), g^{2} \neq 1$. Then

(i) $g$ has a single eigenvalue $\Leftrightarrow g \in \psi\left(S^{3} \times 1\right)$,

(ii) $g$ has a pair of conjugate eigenvalues $\Leftrightarrow g \in \psi\left(1 \times S^{3}\right)$. 
We have the following commutative diagram

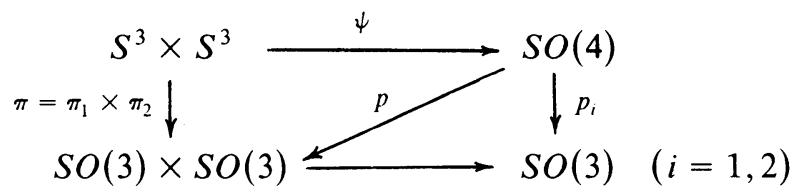

with the vertical maps being the obvious projections.

Proposition 5.2. Assume that $G$ is a finite group.

(i) If $g h g^{-1}=h^{-1} \neq h$ for some $g, h \in G$, then $p_{1}(h)=1$.

(ii) If $p_{2}(G)=(1)$, then $G$ is a cyclic group.

(iii) If $p_{2}(G)$ is cyclic, then $G$ is abelian.

(iv) $G$ is abelian if and only if both $p_{1}(G)$ and $p_{2}(G)$ are cyclic.

Proof. If $g h g^{-1}=h^{-1} \neq h$, then $h$ must be a cohomology. By Theorem 4.5, $g$ interchanges two points fixed by $h$ and consequently the corresponding tangent representations of $h$ are equivalent. It follows from Propositions 2.4 and 5.1 that $p_{1}(h)=1$.

Suppose now that $p_{2}(G)=(1)$. If $G$ were not a cyclic group, $p_{1}(G)$ would contain a dihedral group $D$. Then $D^{*}=G \cap p_{1}^{-1}(D)$ would be a binary dihedral group and therefore we would have $\left|p_{1}\left(D^{*}\right)\right| \leqslant 2$ by part (i). Since $p_{1}\left(D^{*}\right)=D, G$ must be a cyclic group.

If $p_{2}(G)$ is cyclic, let $H=G \cap \operatorname{Ker} p_{2}$ and let $g \in G$ be a generator of $G \bmod H$. By (ii), $H$ is a cyclic group. Let $h \in H$ be a generator. We shall show that $H$ is a central subgroup of $G$. Since $G / H$ is cyclic, it will follow that $G$ is abelian.

Suppose that $H$ is not central in $G$. Then, by Corollary $4.2, h$ is not a homology. Hence $h$ has three fixed points and $g$ interchanges two of them. Then $g^{2} h=h g^{2}$ by Corollary 4.6 and therefore $h \rightarrow g h g^{-1}$ is a nontrivial automorphism of $H$ of order 2. Note that if $h_{1} \in H$ then $g h_{1} g^{-1} \neq h_{1}^{-1}$ or else $p_{1}\left(h_{1}\right)=1$ by part (i), and so $\left|h_{1}\right| \leqslant 2$. It follows that $|H|=2^{t} m$ with $m$ odd, $t \geqslant 3$ and that $g h^{m} g^{-1} \neq h^{ \pm m}$. Thus $g h^{m} g^{-1}=h^{m r}$, where $r \equiv \pm 1+2^{t-1} \bmod 2^{t}$.

Since $r \equiv-1+2^{t-1} \bmod 2^{t}$ implies $g h^{2 m} g^{-1}=h^{-2 m}$, we must have $r \equiv 1+$ $2^{t-1} \bmod 2^{t}$. Then $g h^{2 m} g^{-1}=h^{2 m}$. This forces $h^{2 m}$ to be a homology. Otherwise, $h$ and $h^{2 m}$ would have the same fixed points and hence $\left\langle g, h^{2 m}\right\rangle$ would be an abelian group fixing a single point. The $G$-signature theorem applied to $h^{m}$ now gives

$$
\left(\frac{\omega^{x}+1}{\omega^{x}-1}\right)\left(\frac{\omega^{y}+1}{\omega^{y}-1}\right)=1
$$

for some primitive $2^{t}$ th roots of unity $\omega^{x}, \omega^{y}$. This implies $\omega^{x}=-\omega^{y}$. However, $p_{2}(H)=(1)$ and consequently $\omega^{x}=\omega^{y}$ by Proposition 5.1. The contradiction proves that $H$ is a central subgroup.

Finally, to prove (iv) we assume that $G$ is an abelian group. Then each $p_{i}(G)$ is abelian too. So $p_{i}(G)$ is a cyclic group unless $p_{i}(G)=C_{2} \times C_{2}, i=1,2$. Moreover, both $p_{1}(G)$ and $p_{2}(G)$ are, or both are not, cyclic for otherwise $G$ would not be abelian. Suppose that $p_{i}(G)=C_{2} \times C_{2}, i=1,2$. Then $G$ is conjugate in $S O(4)$ to 
either $\psi\langle(i, i),(j, j)\rangle$ or $\psi\langle(i, i),(j, k)\rangle$. Using the standard basis $1, i, j, k=i j$, we have the following matrix representation:

$$
\begin{gathered}
\psi(i, i)=\left[\begin{array}{cccc}
1 & 0 & 0 & 0 \\
0 & 1 & 0 & 0 \\
0 & 0 & -1 & 0 \\
0 & 0 & 0 & -1
\end{array}\right], \quad \psi(j, j)=\left[\begin{array}{cccc}
1 & 0 & 0 & 0 \\
0 & -1 & 0 & 0 \\
0 & 0 & 1 & 0 \\
0 & 0 & 0 & -1
\end{array}\right], \\
\psi(j, k)=\left[\begin{array}{cccc}
0 & -1 & 0 & 0 \\
-1 & 0 & 0 & 0 \\
0 & 0 & 0 & 1 \\
0 & 0 & 1 & 0
\end{array}\right] .
\end{gathered}
$$

Consequently, in either case $M^{(i}$ would have a 1-dimensional component. Since this cannot happen for $G<\mathscr{G}^{+}(M)$, both $p_{i}(G)$ must be cyclic.

Proposition 5.3. Let $H \subseteq S O(4)$ be a closed subgroup. If $p_{1}(H)$ is finite, there exists a finite subgroup $\hat{H} \subseteq H$ such that $p_{1}(\hat{H})=p_{1}(H)$.

Proof. Since $p_{1}(H)$ is finite, the identity component of $H$ is contained in $\operatorname{Ker} p_{1}$. Thus, given $x \in p_{1}(H)$, there exists $\hat{x} \in H$, an element of finite order such that $p_{1}(\hat{x})=x$.

If $\operatorname{dim} H=1$, then $p_{2}(H)=S O(2)$ or $N(S O(2))$. Hence every torsion, finitely generated subgroup of $H$ is finite. Set $\hat{H}=\left\langle\hat{x} \mid x \in p_{1}(H)\right\rangle$.

If $\operatorname{dim} H>1$, then $p_{2}(H)=S O(3)$ and hence $\psi\left(1 \times S^{3}\right) \subseteq H$. It follows that

$$
H=\psi\left(\pi_{1}^{-1} p_{1}(H) \times S^{3}\right) .
$$

Set $\hat{H}=\psi\left(\pi_{1}^{-1} p_{1}(H) \times 1\right)$.

We are now in a position to prove the main result of this section.

TheOREM 5.4. For any reducible group $G<\mathscr{G}^{+}(M), p_{1}(G)$ is conjugate in $S O(3)$ to a subgroup of $S O(2)$.

Proof. Recall that $p_{1}(G)$ is conjugate to a subgroup of $S O(2)$ if and only if it does not have any dihedral subgroup. We shall assume that $p_{1}(G)$ has a dihedral subgroup and derive a contradiction. Passing to a subgroup of $G$, if necessary, we can assume that $p_{1}(G)$ is a dihedral group. By 5.3, we can also assume that $G$ is finite. It follows from Proposition 5.2 that $G$ is a nonabelian group and that $p_{2}(G)$ is noncyclic. Thus $p_{2}(G)$ is either a dihedral or polyhedral group.

By 5.2, it suffices to show that, under the above hypotheses, $G$ would have to contain a dihedral group $D$ with $p_{1}(D)$ a noncyclic group. This is clear in case $p_{2}(G)$ is a dihedral group. We therefore assume that $p_{2}(G)$ is a polyhedral group. Let $G_{2}$ be a 2-Sylow subgroup of $G$. Then $p_{2}\left(G_{2}\right)$ is a dihedral 2-group, and hence $p_{1}\left(G_{2}\right)$ is cyclic. By assumption, $p_{1}(G)=D_{m}$ for some $m$, so $p_{1}\left(g_{2}\right)=C_{2}$ and $m$ is odd.

We claim that $A=\psi(-1,1) \in G$. For if $A \notin G$, then $G_{2} \cong p\left(G_{2}\right)$, and since $p_{2}\left(G_{2}\right)$ contains $C_{2} \times C_{2}, G_{2} \cap p_{2}^{-1}\left(C_{2} \times C_{2}\right)$ would be an abelian group contradicting 5.2. Thus $G$ acts on the 2-sphere $S$ fixed by $A$. Let $H \subseteq G$ be a maximal subgroup acting trivially on $S$. Then $G / H$ is a finite subgroup of $S O(3)$, since the 
only groups acting effectively on $S^{2}$ are the linear ones (see [E1] and references there). By Corollary 4.2, $H$ is a central subgroup. Since $p_{i}(G), i=1,2$, has no center, $p(H)=(1)$, and so $G / H=p(G)$. But $p(G)$ maps onto $p_{2}(G)$, so $p(G)$ is a polyhedral group and the projection $p(G) \rightarrow p_{2}(G)$ is an isomorphism.

Let $K=G \cap \operatorname{Ker} p_{1}$. Then $p(G) / p(K) \cong p_{1}(G)=D_{m}$. Thus $p(G)$ is the octahedral group, $p(K)=C_{2} \times C_{2}$ and $p(G) / p(K)=D_{3}$. Since the extension

$$
1 \rightarrow p(K) \rightarrow p(G) \rightarrow D_{3} \rightarrow 1
$$

is split, $G$ has a dihedral subgroup $D$ such that $p(D)$ is a diagonal copy of $D_{3}$ in $S O(3) \times S O(3)$.

Theorem 5.4 implies that $G$ is contained in $\psi\left(\pi_{1}^{-1}(S O(2)) \times S^{3}\right)$, which is the copy of the unitary group $U(2)$ we have been looking for.

To conclude this section we give two applications.

Proposition 5.5. Every simple nonabelian group $H<\mathscr{G}^{+}(M)$ is irreducible.

Proof. Suppose $H$ has a fixed point. Since $H$ has no proper normal closed subgroup, $p_{1}(H)=1$ by Theorem 5.4. Thus $H \cong p_{2}(H)$, and so $H$ is either $S O(3)$ or the icosahedral group. In either case it contains, contrary to Proposition 5.2, a copy of $C_{2} \times C_{2}$ such that $p_{2}\left(C_{2} \times C_{2}\right)$ is not cyclic.

Proposition 5.6. Let $H<\mathscr{G}^{+}(M)$ and let $h \in H$ be a homology. Then $Z_{H}(h)=$ $\{x \in H: x P=P\}$, where $P$ is the isolated fixed point of $h$.

Proof. If $x \in Z_{H}(h)$, then clearly $x P=P$. Conversely, suppose that $x P=P$. The tangent representation of $\langle h, x\rangle$ at $P$ is complex and $h$ has a single eigenvalue. Consequently, $h$ commutes with all elements of $\langle h, x\rangle$.

\section{Imprimitive groups.}

Definition 6.1. An irreducible group $G<\mathscr{G}^{+}(M)$ is called imprimitive, if it has a normal reducible subgroup $K \neq(1)$. If there is no subgroup with this property, $G$ is said to be primitive.

We assume throughout this section that $G$ is an imprimitive group.

Note that any normal reducible subgroup $K \subseteq G$ is necessarily abelian and has three isolated fixed points. Otherwise, according to Theorem $4.5, K$ would have a single isolated fixed point, and so would $G$. Thus the action of $G$ on $M^{K}$ defines a group homomorphism

$$
\varphi_{K}: G \rightarrow S_{3}=\left\langle u, v \mid u^{3}=v^{2}=1, v u v=u^{2}\right\rangle .
$$

Clearly, $K$ is a maximal normal reducible subgroup if and only if $K=\operatorname{Ker} \varphi_{K}$. Moreover, $\varphi_{K}(G)$ contains the 3 -cycle $u$, since $G$ is irreducible. Thus, for a maximal $K, G / K$ is isomorphic to a cyclic or dihedral group, of order 3 or 6 , respectively.

DEFINITION 6.2. Let $R(G) \subseteq G$ be a maximal normal reducible subgroup and let $T(G) \subseteq R(G)$ be a maximal toral group, that is, a group isomorphic to $J \times J$ for some $J \subseteq S O(2)$. We denote by $S(G)$ the unique subgroup of $G$ that contains $R(G)$ as a subgroup of index 3 .

It follows that $T(G)$ is a normal reducible subgroup of $G$ while $S(G)$ is an irreducible subgroup of index $\leqslant 2$. 
Proposition 6.3. $R(G)$ is a unique maximal normal reducible subgroup of $G$ if and only if $S(G)$ is a nonabelian group. If $S(G)$ is abelian, $G$ has precisely four normal reducible subgroups, each of order 3.

Proof. Assume first that $S(G)$ is abelian. By Proposition 3.2, $S(G)$ is an elementary abelian group of order 9 . Therefore, it has four reducible subgroups, each of order 3. If $[G: S(G)]=2$, the element of order 2 does not commute with $R(G)$ by Corollary 4.6. It follows that $G$ has the following presentation:

$$
\left\langle x, u, v \mid x^{3}=u^{3}=v^{2}=1, x u=u x, v x v=x^{2}, v u v=u^{2}\right\rangle
$$

and, in particular, each cyclic subgroup of $S(G)=\langle x, u\rangle$ is normal in $G$. Also, $G$ has no other normal reducible subgroup.

Assume now that $S(G)$ is a nonabelian group. Suppose also $K \subseteq G$ is a maximal normal reducible subgroup. We shall show $K=R(G)$. Notice first that since $S(G)$ is irreducible, it must have order $>9$. Indeed, every nonabelian group of order $3 k \leqslant 9$ is isomorphic to $D_{3}$ and as such must have a fixed point. Consequently, $|R(G)|>3$. Since $R(G)$ is abelian, $\varphi_{K}(R(G))$ has order $\leqslant 3$. It follows that $K \cap R(G) \neq(1)$. Thus $L=K \cap R(G)$ is a normal reducible subgroup of $G$. As noted before, $L$ has three fixed points. Since $M^{K} \cup M^{R(G)} \subseteq M^{L}$, we obtain $M^{K}=M^{R(G)}$. Hence $K \cdot R(G) \subseteq G$ is a normal reducible subgroup having the same fixed point set. Thus $K=K \cdot R(G)=R(G)$ by maximality.

Proposition 6.4. Given $g \in S(G)-R(G)$, then $g^{3}=1$. Hence

$$
1 \rightarrow R(G) \rightarrow S(G) \rightarrow C_{3} \rightarrow 1
$$

is a split extension.

Proof. Let $g \in S(G)-R(G)$. Since $\varphi_{R(G)}\left(g^{3}\right)=1, g^{3} \in R(G)$. If $g^{3} \neq 1$, it would have at least one isolated fixed point. Then both $R(G)$ and $g$ would have to fix this point.

Proposition 6.4 allows us to identify the 3-cycle $u \in \varphi(G)$ (here $\varphi=\varphi_{R(G)}$ ) with an element of $G$, also denoted by $u$.

Proposition 6.5. $T(G)$ contains all homologies in $R(G)$.

Proof. If $g \in R(G)$ is a homology, then $\langle g\rangle \cap u\langle g\rangle u^{-1}=(1)$, since $u$ does not fix the isolated fixed point of $g$. Thus $\left\langle g, u g u^{-1}\right\rangle=\langle g\rangle \times\left\langle u g u^{-1}\right\rangle$ and consequently $g \in T(G)$.

To determine the group structure of $G$ we are going to study the action of $\varphi(G)$ on $R(G)$. This is easy for $S(G)$ an abelian group. In fact, the proof of Proposition 6.3 even provided a presentation of $G$ in this case. The nonabelian case is more complicated and requires some preliminary work.

Proposition 6.6. Suppose $K \subseteq G$ is a normal cyclic subgroup of order $n$. The $\varphi(G)$-module structure of $K$ is given by $u: x \rightarrow x^{r}, v: x \rightarrow x^{-1}$, where $x \in K$ is a generator and $r^{2}+r+1 \equiv 0 \bmod n$. Moreover, $n$ is an odd number nondivisible by 9 and $G=S(G)$ unless $n=3$. 
Proof. Since $u^{3}=1, u x u^{-1}=x^{r}$ for some $r$ such that $r^{3} \equiv 1(\bmod n)$.

If $r \equiv 1 \bmod n, S(G)$ is abelian, hence isomorphic to $C_{3} \times C_{3}$. Consequently, $n=3$.

If $\operatorname{gcd}(3, n)=1$, then $r \neq 1 \bmod m$ for any $m \mid n, m>1$. Thus $\operatorname{gcd}(r-1, n)=1$, whence $r^{2}+r+1 \equiv 0 \bmod n$.

Suppose now that $n=9 k$ for some $k \geqslant 1$. It follows then from $r^{3} \equiv 1 \bmod 9$, $r \neq 1 \bmod 9$ that $r \equiv 1 \pm 3 \bmod 9$ and $u x^{k}$ has order 9 . This, however, contradicts Proposition 6.4. We therefore conclude that $n \neq 0 \bmod 9$ and $r^{2}+r+1 \equiv 0$ $\bmod n$. Also, since $K$ contains no homology, $n \equiv 1 \bmod 2$.

Finally, assume $[G: S(G)]=2$. Let $h \in G$ represent $v \in \varphi(G)$. Then, given $y \in K, \quad y \neq 1,\langle y, h\rangle$ fixes a single point because $M^{y}=M^{K}$. It follows from Corollary 4.6 that $\langle y, h\rangle$ is a nonabelian group for each $y \in K$. This proves $h x h^{-1}=x^{-1}$.

Hence the tangent representations of $K$ at fixed points, which get interchanged by $v$, are equivalent. Thus, by Proposition $2.4, x$ has two conjugate eigenvalues at the third fixed point. The same argument applies to the tangent representations of $K$ at fixed points interchanged by $u v u^{-1}$. It follows that $x$ has the same two conjugate eigenvalues, say $\left\{\omega, \omega^{-1}\right\}$, at all three fixed points. Since $u x u^{-1}=x^{r}$, we must also have

$$
\left\{\omega, \omega^{-1}\right\}=\left\{\omega^{r}, \omega^{-r}\right\}=\left\{\omega^{r^{2}}, \omega^{-r^{2}}\right\} .
$$

This together with $r^{2}+r+1 \equiv 0 \bmod n$ implies that $r \equiv 1 \bmod n$. Consequently, $n=3$.

Given a prime $p$ and integer $f \geqslant 0$, let $R(G)_{p}^{f}$ and $T(G)_{p}^{f}$ denote the $p^{f}$-torsion subgroups of $R(G)$ and $T(G)$, respectively. Let $R(G)_{p}=U_{f} R(G)_{p}^{f}$ and $T(G)_{p}=$ $U_{f} T(G)_{p}^{f}$.

Proposition 6.7. Given coordinate homologies $x, y \in T(G)_{p}^{f}$, the $\varphi(G)$-module structure of $T(G)_{p}^{f}$, is determined by the matrices

$$
U=\left[\begin{array}{ll}
0 & -1 \\
1 & -1
\end{array}\right], \quad V=\left[\begin{array}{ll}
0 & 1 \\
1 & 0
\end{array}\right]
$$

Proof. According to Proposition 3.4, every homology in $T(G)_{p}^{f}$ belongs to $\langle x\rangle$, $\langle y\rangle$ or $\langle z=x y\rangle$ and each point fixed by $T(G)$ is an isolated fixed point of $x, y$ or $z$. Hence, given $g \in G$ and a homology $h \in T(G)_{p}^{f}$, whether $g h g^{-1}$ belongs to $\langle x\rangle$, $\langle y\rangle$ or $\langle z\rangle$ can be determined from the action of $g$ on $M^{T(G)}$, and this depends only on $\varphi(g)$. Thus we can assume, without loss of generality, that $u\langle x\rangle u^{-1}=\langle y\rangle$, $u\langle y\rangle u^{-1}=\langle z\rangle$.

We have then $u x u^{-1}=y^{s}, u y u^{-1}=z^{t}$ for some $s, t, \operatorname{gcd}(s t, m)=1, m=|x|$. Thus $u^{3}=1$ implies $s^{3} \equiv 1 \bmod m, s \equiv-t \bmod m$ and $(u x)^{3}=x^{1-s^{2}} y^{s-s^{2}}$. Since $(u x)^{3}$ $=1$ by Proposition 6.4, $s \equiv 1 \bmod m$. Thus

$$
u x u^{-1}=y \text { and } u y u^{-1}=x^{-1} y^{-1},
$$

as claimed.

Assume now that $[G: S(G)]=2$. Let $h \in G$ represent $v \in \varphi(G)$. We can assume that $h\langle x\rangle h^{-1}=\langle y\rangle$. Thus $h x h^{-1}=y^{r}$ for some $r, \operatorname{gcd}(r, m)=1$. Since $v^{2}=1$, $h\langle y\rangle h^{-1}=\langle x\rangle$ and $h\langle z\rangle h^{-1}=\langle z\rangle$, we see that $h z h^{-1}=h x y h^{-1}=x^{1 / r} y^{r}$. But 
$h z h^{-1}=z$ by Corollary 4.2, so $y^{\prime r}=y$. Hence

$$
h x h^{-1}=y \text { and } h y h^{-1}=x .
$$

Suppose now that $R(G)_{p}^{f}=C_{n} \times C_{m}$, where $n=p^{f}$ and $m \mid n$. Then any element $g \in R(G)_{p}$ of order $n$ generates $R(G)_{p}^{f} \bmod T(G)_{p}^{f}$.

Proposition 6.8. Assume $n>m>1$. Then $\langle g\rangle \subseteq G$ is a normal subgroup unless $p=3$ and $n=3 \mathrm{~m}$. In the exceptional case there are coordinate homologies $x, y \in$ $T(G)_{3}^{f}$ such that $g^{3}=x y^{-1}$ and $u g u^{-1}=g^{r} y$ for some $r \equiv 1 \bmod m$. Furthermore, $G=S(G)$ unless $p=3, n=3 m, r \equiv 1 \bmod n$ and $h g h^{-1}=g^{-1}$ for some $h \in G-$ $S(G)$.

Proof. Let $V$ be a tangent representation of $R(G)_{p}^{f}$. Since $R(G)_{p}^{f}$ is abelian, $V=V_{1} \oplus V_{2}$. Let $\chi_{i}: R(G)_{p}^{f} \rightarrow S^{1}$ be the character corresponding to $V_{i}(i=1,2)$. Then either $\chi_{1}(g)$ or $\chi_{2}(g)$ is a primitive $n$th root of unity. Assume, without loss of generality, that $\chi_{1}(g)=\omega$ is a primitive $n$th root. Then $\chi_{2}(g)=\omega^{a}$ for some $a$, and Proposition 6.5 implies that $\operatorname{gcd}(a, p)=1$.

There are also $x, y \in R(G)_{p}^{f}$ such that $\chi_{2}(x)=\chi_{1}(y)=1$ and $\chi_{1}(x)=\chi_{2}(y)=$ $\omega^{k}$, where $k=n / m$. It follows from 3.4 and 2.3 that $x, y$ are coordinate homologies for $T(G)_{p}^{f}$. Thus by 6.7, the action of $\varphi(G)$ on $T(G)_{p}^{f}$ is given by

$$
u x u^{-1}=y, \quad u y u^{-1}=x^{-1} y^{-1} \quad \text { and } \quad h x h^{-1}=y,
$$

where $h \in G$ is any element representing $v$ (provided $v \in \varphi(G)$, of course).

Notice first that $g^{k} \in T(G)_{p}^{f}$. In fact, $\left(\chi_{1}\left(g^{k}\right), \chi_{2}\left(g^{k}\right)\right)=\left(\omega^{k}, \omega^{k a}\right)$, so $g^{k}=x y^{a}$. It follows that $R(G)_{p}^{f}=\langle g\rangle \times\langle y\rangle$ and $u g u^{-1}=g^{r} y^{s}$ for some $r, s, \operatorname{gcd}(r, p)=1$. Thus $u^{3}=1$ implies

$$
g=g^{r^{3}} x^{s-r s} y^{r^{2} s-r s}, \text { and } s(r-1) \equiv 0 \quad \bmod m .
$$

Second, $u g^{m} u^{-1}=g^{m r}$, so $k \neq 0 \bmod 9$ by Proposition 6.6.

Suppose now $s \neq 0 \bmod m$. Together with $(*)$, this implies $r \equiv 1 \bmod p$. Thus $\left\langle g^{m}, u\right\rangle$ has a center and therefore $p=3$ by Proposition 3.2. (In particular, $\langle g\rangle$ is normal in $S(G)$ for $p \neq 3$.) But $k \neq 0 \bmod 9$, so $k=p=3$. Let $q$ denote the order of $s \bmod m$. Thus $u g^{4} u^{-1}=g^{q r}$, so $n / q=3$ by Proposition 6.6. Hence $q=m$ and $s$ is a unit $\bmod m$. This shows $r \equiv 1 \bmod m$. Moreover,

$$
(u g)^{3}=g^{r^{2}+r+1} x^{-s} y^{r s} .
$$

Hence

$$
\chi_{1}(u g)^{3}=\chi^{r^{2}+r+1-3 s} \quad \text { and } \quad \chi_{2}(u g)^{3}=\omega^{a\left(r^{2}+r+1\right)+3 r s} .
$$

But $(u g)^{3}=1$ by Proposition 6.4, so

$$
\begin{gathered}
r^{2}+r+1 \equiv 3 s \quad \bmod n, \\
a\left(r^{2}+r+1\right)+3 r s \equiv 0 \quad \bmod n .
\end{gathered}
$$

Since $r \equiv 1 \bmod m$, we also have $3 r s \equiv 3 s \bmod n$ and $r^{2}+r+1 \equiv 3 \bmod n$. Consequently,

$$
\begin{gathered}
r^{2}+r+1 \equiv 3 s \equiv 3 \bmod n, \\
3(a+1) \equiv 0 \quad \bmod n .
\end{gathered}
$$


Since $n=3 m$, we see that $s \equiv 1 \bmod m$ and $a \equiv-1 \bmod m$. This shows that

$$
g^{3}=x y^{-1}, \quad u g u^{-1}=g^{r} y
$$

and completes the proof in the case $G=S(G)$. We also note that Proposition 6.6 implies $p>2$, because $\langle g\rangle$ is normal in $S(G)$ for $p \neq 3$.

Suppose finally that $[G: S(G)]=2$. Then $h g h^{-1}=g^{t} y^{s_{1}}$ for some $t, \operatorname{gcd}(t, p)=1$. Since $v^{2}=1, g=g^{t^{2}} x^{s_{1}} y^{t s_{1}}$. This implies $s_{1} \equiv 0 \bmod m$ and $h g h^{-1}=g^{t}$. Consequently, $u g u^{-1} \notin\langle g\rangle$ or else $\langle g\rangle \subseteq G$ would be a normal subgroup. Since $g$ has order $n \neq 3$, the latter possibility can be ruled out by Proposition 6.6. It follows that $p=3, n=3 m$ and $u g u^{-1}=g^{r} y$ with $r \equiv 1 \bmod m$. (In particular, $G=S(G)$ for $p \neq 3$.) Moreover, $g^{t} \neq g$ by Corollary 4.6 , so $h g h^{-1}=g^{-1}$.

Therefore the tangent representations of $g$ at fixed points interchanged by $h$ are equivalent. Since $h x h^{-1}=y, h$ interchanges the isolated fixed points of $x$ and $y$. The tangent representation of $g$ at the isolated fixed point of $x y$ is given by $\left(\chi_{1}(g), \chi_{2}(g)\right)=\left(\omega, \omega^{a}\right)$. Thus $\omega^{a}=\omega^{-1}$ by Proposition 2.4. Since $u g u^{-1}=g^{r} y$ and $u^{2} g u^{-2}=g^{r^{2}} x^{-1}$, the other two tangent representations of $g$ correspond to $\left(\omega^{r}, \omega^{-r+3}\right)$ and $\left(\omega^{r^{2}-3}, \omega^{-r^{2}}\right) \stackrel{(* *)}{=}\left(\omega^{-r-1}, \omega^{r-2}\right)$. Thus the only possibility for these representations to be equivalent is $r \equiv 1 \bmod n$.

Corollary 6.9. $R(G)_{2}=T(G)_{2}$.

Proof. Propositions 6.8 and 6.6 imply that $R(G)_{2} \subseteq T(G)$.

Proposition 6.10. $1 \rightarrow R(G) \rightarrow G \rightarrow \varphi(G) \rightarrow 1$ is a split extension.

Proof. $G$ corresponds to an element $\sigma \in H^{2}(\varphi(G) ; R(G))$. We have to show $\sigma=0$. According to Proposition 6.4 and [ $\mathbf{B r}$, p. 84], it suffices to consider the case $[G: S(G)]=2$ and show that $\operatorname{Res} \sigma=0$ in $H^{2}(\langle v\rangle ; R(G))=H^{2}\left(\langle v\rangle ; R(G)_{2}\right)$.

By 6.9 and 6.7, there are coordinate homologies $x, y \in R(G)_{2}^{f}$ such that $h x h^{-1}=y$ and $h y h^{-1}=x$, where $h \in G_{2}^{f}$ represents $v \in \varphi(G)$ for some $f \gg 0$. Since $G_{2}^{f}$ is a nonabelian 2-group by Corollary 4.6, it follows from Proposition 4.8 that the center $Z\left(G_{2}^{f}\right)$ is generated by a single homology. Since $z=x y$ is in the center, $Z\left(G_{2}^{f}\right)=\langle z\rangle$. Also, $h^{2} \in Z\left(G_{2}^{f}\right)$, so $h^{2}=z^{k}$ for some $k$. Thus, if we define $w=h x^{-k}$, then

$$
w^{2}=\left(h x^{-k}\right)^{2}=y^{-k} h^{2} x^{-k}=1 .
$$

This shows $\operatorname{Res} \sigma=0$.

THEOREM 6.11. Every imprimitive group $G<\mathscr{G}^{+}(M)$ is isomorphic to an imprimitive subgroup of $P U(3)$.

Proof. According to $6.10, G$ is isomorphic to the semidirect product $R(G) \rtimes$ $\varphi(G)$. The $\varphi(G)$-action on $R(G)$ is completely determined by the action on $R(G)_{p}$ for every $p$. This, in turn, was described in Propositions 6.6-6.8. As demonstrated in Appendix 2, each such group has a faithful imprimitive representation in $P U(3)$.

7. Primitive groups having normal imprimitive subgroups. Let $G<\mathscr{G}^{+}(M)$ be a primitive group. We assume that $G$ has a normal imprimitive subgroup $G_{1}$. Then $G$ operates by conjugation on the set of maximal normal reducible subgroups in $G_{1}$. As 
we have seen, every imprimitive group has either one or four such subgroups. Thus $G_{1}$ must have four, since otherwise $G$ would not be primitive. It follows from Proposition 6.3 that $S\left(G_{1}\right)$ is an elementary abelian group of order 9. Moreover, $S\left(G_{1}\right)$ is a normal 3-Sylow subgroup of $G_{1}$. Hence $S\left(G_{1}\right)$ is also a normal subgroup of $G$. Henceforth $S\left(G_{1}\right)$ will be denoted by $H$.

We are interested in the action of $G$ on $H$. Notice first that $Z_{G}(H)=H$, as $H$ is a maximal abelian group by Proposition 3.2. Thus $G / H$ is isomorphic to a subgroup of $\operatorname{Aut}(H)=G L(2,3)$. In particular, $G$ is a finite group.

Proposition 7.1. $1 \rightarrow H \rightarrow G \stackrel{4}{\rightarrow} G / H \rightarrow 1$ is a split extension.

Proof. $G$ corresponds to an element $\sigma \in H^{2}(G / H ; H)$. By [Br, p. 84], it suffices to show that Res $\sigma=0$ in $H^{2}(q(P) ; H)$ for each Sylow subgroup $P \subseteq G$. Since $H$ has no $p$-torsion for $p \neq 3$, we have to consider only the 3-Sylow subgroup. We can assume that $q(P) \neq(1)$. Since $G L(2,3)$ has a 3-Sylow subgroup of order $3,[P: H]$ $=3$. Then $P$ must be a nonabelian irreducible group.

Suppose there is an element $z \in P$ of order 9 . Then $\langle z\rangle$ is normal in $P$, so $P$ is an imprimitive group with $R(P)=\langle z\rangle$. But, according to Proposition 6.6, 9 cannot divide the order of $R(P)$. This proves that all elements of $P$ have order 3 and, in particular, Res $\sigma=0$.

As a subgroup of $\operatorname{Aut}(H), G / H$ acts on the set of cyclic subgroups of $H$. Given two generators $x, y \in H$, each $g \in \operatorname{Aut}(H)$ determines a permutation of the letters $\langle x\rangle,\langle y\rangle,\langle x y\rangle$ and $\left\langle x^{2} y\right\rangle$. Hence there is a well-defined group homomorphism $p: G L(2,3) \rightarrow S_{4}$. These groups have orders 48 and 24 , respectively. Since $A=$ $\left[\begin{array}{rr}-1 & 0 \\ 0 & -1\end{array}\right]$ is the only nontrivial automorphism leaving fixed all four letters, we see that $p$ is onto and $S L(2,3)=p^{-1}\left(A_{4}\right)$.

We want to identify the subgroup $L \subseteq S_{4}$ of all permutations corresponding to elements from $G$. Note that the hypothesis about $G$ being primitive, stated in terms of $L$, guarantees that no letter remains fixed under all permutations from $L$.

Proposition 7.2. The only permutation $\alpha \in L$ that leaves two letters fixed is $\alpha=1$.

Proof. Suppose $\alpha \neq 1$. We can assume, without loss of generality, that $\alpha\langle x\rangle=$ $\langle x\rangle, \alpha\langle y\rangle=\langle y\rangle$ and $\alpha\langle x y\rangle=\left\langle x^{2} y\right\rangle$. Let $g \in G$ represent $\alpha$. Then either $g x g^{-1}$ $=x^{2}$ or $g y g^{-1}=y^{2}$.

Suppose that $g x g^{-1}=x^{2}$. Then $g\langle x y\rangle g^{-1}=\left\langle x^{2} y\right\rangle$ implies $g y g^{-1}=y$. Consequently, $\langle x, g\rangle$ is a normal subgroup of $\langle H, g\rangle$. By Theorem $4.5,\langle x, g\rangle$ has a single fixed point, and so does $\langle H, g\rangle$, a contradiction.

Proposition 7.3. $A \in G L(2,3)$ is the only element of order 2 contained in $G / H$.

Proof. Notice first that $L$ must contain an element of order 2, for otherwise it would fix a letter. Let $g \in G$ represent such an element. Then $q\left(g^{2}\right) \in \operatorname{Ker} p$.

Suppose $q\left(g^{2}\right)=1$. By assumption, there exists $u \in H$ such that $g\langle u\rangle g^{-1} \neq\langle u\rangle$. Then $u=g v g^{-1}$, where $v=g u g^{-1}$. This implies that $g(u v) g^{-1}=u v \notin\left\langle u^{2} v\right\rangle$ and contradicts Proposition 7.2. Hence $q\left(g^{2}\right) \neq 1$ and therefore $q\left(g^{2}\right)=A$. 
Proposition 7.4. L does not contain a 4-cycle.

Proof. Suppose it does contain a 4-cycle and let $g \in G$ be a representative. By Proposition 7.3, $q(g) \in G / H$ is an automorphism of order 8. Since all cyclic subgroups of order 8 are conjugate in $G L(2,3)$, we can assume that $q(g)=\left[\begin{array}{cc}1 & -1 \\ 1 & 1\end{array}\right]$. Thus $K=\langle H, g\rangle$ is isomorphic to $H \rtimes C_{8}$ with the given action of $C_{8}$ on $H$.

We claim that $H^{3}(K)=0$. Since the 2-Sylow subgroup of $K$ is cyclic and $H^{3}$ vanishes on cyclic groups, it suffices to show that $K / H$ acts nontrivially on $H^{3}(H)=\mathbf{Z}_{3}$ (see [Br, p. 84]). Since

$$
H^{3}(H)=\operatorname{Tor}\left(H^{3}(H), \mathbf{Z}_{3}\right) \cong H^{2}\left(H, \mathbf{Z}_{3}\right) / H^{2}(H) \otimes \mathbf{Z}_{3}
$$

by the universal coefficient theorem, the algebra structure of $H *\left(H, \mathbf{Z}_{3}\right)$ can be used to compute the action on $H^{3}(H)$.

Let $X$ be a principal $S O(2)$-bundle over $M$ such that $c_{1}(X)=\alpha \in H^{2}(M)$. Then the cohomology Serre spectral sequence of $X \rightarrow M$ is isomorphic to that of $S^{5} \rightarrow$ $\mathrm{C} P^{2}$ and therefore $X$ is an integral cohomology 5-sphere. Since $H^{1}(M)=0$, it is a consequence of [HY] that $H^{3}(K)$ contains the only obstruction to lifting the action of $K$ from $M$ to $X$.

Thus in our situation the action of $K$, hence that of $H$, can be lifted to $X$. Since each element $h \in H, h \neq 1$ has order 3, it follows from [B, Theorem III.7.11] that $X^{h}$ is a connected mod 3 cohomology sphere. Suppose $X^{h} \neq \varnothing$ for some $h \in H$. The bundle projection $r: X \rightarrow M$ maps $X^{h}$ into $M^{h}$. Since $M^{h}$ consists of three isolated points, we see that $r\left(X^{h}\right)$ must be a single point. Furthermore, $H X^{h}=X^{h}$ implies $\operatorname{Hr}\left(X^{h}\right)=r\left(X^{h}\right)$. Hence $H$ has a fixed point, a contradiction. It follows that $H$ acts freely on $X$. It is well known, however, that an abelian group acting freely on a cohomology sphere is cyclic (see [B, Theorem III.8.1]). The contradiction proves that $L$ cannot contain a 4 -cycle.

It follows from Propositions 7.2 and 7.4 that $L \subseteq A_{4}$. The alternating group $A_{4}$ has, up to conjugation, only three fixed point free subgroups. These are $C_{2}, C_{2} \times C_{2}$ and $A_{4}$. Thus, by Proposition 7.3, G/H= $p^{-1}(L)$ is isomorphic to $C_{4}$, the quater nion group $Q$ or $S L(2,3)$. This together with Proposition 7.1 implies the following.

THEOREM 7.5. $G$ is isomorphic to $H \rtimes S L(2,3)$ or to one of its subgroups $H \rtimes C_{4}$, $H \rtimes Q$.

Remark. Suppose that $G^{1}<\mathscr{G}^{+}(M)$ is a primitive group containing $G$ as a normal subgroup. Since $H$ is a characteristic subgroup of $G$, it is also normal in $G^{1}$. It follows that $G^{1}$ is isomorphic to a subgroup of the Hessian group.

8. Simple primitive groups of finite order. We wish to determine all finite primitive groups that have no normal imprimitive subgroup. They all turn out to be simple.

First we need the following.

DEFINITION 8.1. A finite nonabelian group $H$ is said to be geometric, if

(i) its $p$-Sylow subgroups are abelian for $p>3$,

(ii) its nonabelian Sylow subgroups have a cyclic center,

(iii) $p$-rank $H \leqslant 2$ for every prime $p$, and

(iv) $N_{H}(K) / Z_{I I}(K)$ is isomorphic to a subgroup of $D_{3}$ for each abelian subgroup $K \subseteq H, K \neq C_{3} \times C_{3}$. 
Proposition 8.2. Every simple geometric group is isomorphic to PSL $(2,3)$, $\operatorname{PSL}(2,7), \operatorname{PSL}(2,9)$ or PSL $(3,3)$.

Proof. It is known (see [G, Theorem 1.86]) that every finite simple group of 2-rank at most 2 is isomorphic to one from the following list:

(i) $\operatorname{PSL}\left(2, p^{r}\right), p^{r}>3, p$ odd.

(ii) $\operatorname{PSL}\left(3, p^{r}\right), p$ odd,

(iii) $\operatorname{PSU}\left(3, p^{2 r}\right), p$ odd or $p^{r}=4$,

(iv) $A_{7}$,

(v) $M_{11}$.

These groups and their subgroup structures are well understood. In particular, the only geometric groups on this list are those as claimed. The reader can consult $[\mathbf{H}]$ (especially pp. 185, 191, 204, 242) and [CM].

Proposition 8.3. Suppose $G$ is a subgroup of $\operatorname{Aut}(H)$, where $H=\operatorname{PSL}(2, p)$, $p=5.7$ or 9. If $G$ is geometric, then either $G=H$ or $G=P G L(2,9)$.

Proof. For $H=\operatorname{PSL}(2, p)$ and $p=5$ or 7 , Aut $H)=\operatorname{PGL}(2, p)$ by [SW]. Since $G \neq(1)$ and $H$ is simple, $H \subseteq G$. Hence either $G=H$ or $G=\operatorname{Aut}(H)$. But $P G L(2, p), p \geqslant 5$ is not geometric since $N_{G}(P) / Z_{G}(H)=C_{p-1}$ for its $p$-Sylow subgroup $P$ (see [H, p. 185]). It follows that $G=H$ in this case.

For $H=P S L(2,9) \cong A_{6}\left(\right.$ see [H, p. 199]), we have $\operatorname{Aut}(H)=\operatorname{Aut}\left(S_{6}\right) \cong S_{6} \rtimes C_{2}$ (see [R. Theorem 7.8]). Since $H \subseteq G$ (again by simplicity), there are only five possibilities for $G$ : $H$, Aut $H)$ and the three subgroups of index 2 in $\operatorname{Aut}(H)$. To identify the intermediate subgroups, it suffices to find three nonisomorphic groups of order 720, each containing $A_{6}$ as a unique proper normal subgroup. Such groups do exist; they are $S_{6}, \operatorname{PGL}(2,9)$ and $M_{10}$. They are indeed nonisomorphic because they have nonisomorphic 2-Sylow subgroups: $D_{4} \times C_{2}, D_{8}$ and the quasidihedral group.of order 16, respectively (see [R, Theorem 9.49]). In particular, $S_{6}$ and Aut $(H)$ have 2-rank 3 and hence are not geometric. Also the Mathieu group $M_{10}$ is not geometric. This can be seen as follows.

Let $P \subseteq H$ be a 5-Sylow subgroup. Label $S_{6}, P G L(2,9)$ and $M_{10}$ as $K_{1}, K_{2}, K_{3}$, respectively. Let $N_{i}(P)$ and $Z_{i}(P)$ denote respectively the normalizer and centralizer of $P$ in $K_{i}$. By [H, pp. 187, 204],

$$
\begin{aligned}
& Z_{1}(P)=P, \quad N_{1}(P) / Z_{1}(P)=C_{4}, \\
& Z_{2}(P)=P \times C_{2}, \quad N_{2}(P) / Z_{2}(P)=C_{2}, \\
& Z_{H}(P)=P, \quad N_{H}(P) / Z_{H}(P)=C_{2} .
\end{aligned}
$$

Since $N_{1}(P) \cap N_{2}(P)=N_{H}(P)$, we deduce from the above that

$$
Z_{3}(P)=P, \quad N_{3}(P) / Z_{3}(P)=C_{4} .
$$

This shows that $M_{10}$ is not geometric and hence either $G=H$ or $G=P G L(2,9)$.

LEMMA 8.4. If $H<\mathscr{G}^{+}(M)$ is a finite simple primitive group, then $H$ is isomorphic to $\operatorname{PSL}(2, p)$ with $p=5,7$ or 9 .

Proof. It follows from the previous sections that $H$ is geometric. Hence $H$ is isomorphic to one of the groups in Proposition 8.2. To prove the lemma, we have to show that $H \neq \operatorname{PSL}(3,3)$. 
Suppose $H \cong \operatorname{PSL}(3,3)$. It is known (see [H, p. 189]) that $S$, the 2-Sylow subgroup of $H$, normalizes some 3-Sylow subgroup $P . P$ is a nonabelian group of order 27, so its center $Z(P)$ has three fixed points by Proposition 4.8. Since $Z(P)$ is also a normal subgroup of $S P$, the latter is an imprimitive group. Let $R(S P)$ denote a maximal normal reducible subgroup of $S P$. Since $R(S P)$ is abelian and $S$ is a quasidihedral group of order 16, it follows from Proposition 4.7 that $R(S P) \cap S$ has index 2 in $S$. In particular, $R(S P) \cap S$ is not a toral group, which contradicts Corollary 6.9.

Proposition 8.5. Let $G<\mathscr{G}^{+}(M)$ and let $H \subseteq G$ be a primitive subgroup. Then $Z_{G}(H)=(1)$.

Proof. Suppose there is $g \in Z_{G}(H), g \neq 1$. Then $H$, acting on $M^{g}$, must be either reducible or imprimitive.

THEOREM 8.6. If $G<\mathscr{G}^{+}(M)$ is a finite primitive group having no normal imprimitive subgroup, then $G$ is simple, hence isomorphic to $\operatorname{PSL}(2,5), \operatorname{PSL}(2,7)$ or $\operatorname{PSL}(2,9)$.

Proof. Suppose $G$ is not simple. By assumption, its proper normal subgroups are primitive too. As noted in the end of $\$ 7$, none of these normal subgroups have a normal imprimitive subgroup, for this would force $G$ to have the same property. Thus, if the nonsimple primitive group $G$ exists at all, it must contain a minimal nonsimple primitive subgroup in the sense that all its proper normal subgroups are simple. Consequently, we may assume that $G$ itself is such a minimal group.

Let $H \subseteq G$ be a normal simple subgroup. By Lemma 8.4,H=PSL $(2, p)$ with $p=5,7$ or 9 . Conjugation by elements of $G$ defines a group homomorphism $G \rightarrow \operatorname{Aut}(H)$. By Proposition 8.5, this homomorphism is one-to-one, so $G$ can be identified with a subgroup of $\operatorname{Aut}(H)$. Hence, by Proposition 8.3, $G=P G L(2,9)$.

Let $P \subseteq G$ be a 3-Sylow subgroup. According to [H, p. 185], $Z_{G}(P)=P$ and $Z_{G}(P) / P=C_{8}$. Since $P$ is abelian, Proposition 4.7 implies that $P$ is irreducible. It follows from Corollary 6.9 that $N_{G}(P)$ is not imprimitive. Hence $N_{G}(P)$ is a primitive group and $P$ is its normal imprimitive subgroup. However, $N_{G}(P)$ is not isomorphic, to a subgroup of the Hessian group, which contradicts Theorem 7.5.

\section{Compact primitive groups.}

Lemma 9.1. Let $G<\mathscr{G}^{+}(M)$ be a nonabelian connected group. Then $G=S O(3)$, $S U(2), U(2)$ or $P U(3)$.

Proof. If rank $G=1$, then $G=S O(3)$ or $S U(2)$. Otherwise, rank $G=2$. Since $H_{1}(M)=0$, it is a consequence of $[\mathbf{O R}]$ that $\pi_{1}(M)=0$ and, in fact, $M=\mathbf{C} P^{2}$. It follows from $[\mathbf{O}]$ that $G=U(2)$ or $P U(3)$ in this case.

Proposition 9.2. Suppose $G<\mathscr{G}^{+}(M), \operatorname{dim} G>0$, is a primitive group. Then $G=S O(3)$ or $P U(3)$.

Proof. Let $G_{0}$ denote the identity component of $G$. Since $G$ is primitive, $G_{0}$ must be an irreducible and hence nonabelian group. In particular, $G_{0}$ has no central involution. By the lemma, $G_{0}=S O(3)$ or $P U(3)$. 
Suppose that $G_{0}=S O(3)$. The adjoint representation of $G$ defines a homomorphism $h: G \rightarrow S O(3)$. Since $S O(3)$ is simple, we see that $G=\operatorname{Ker} h \times G_{0}$. It follows from Proposition 8.5 that $G=G_{0}$.

If $G_{0}=P U(3)$, let $H$ be the principal isotropy group for the action of $G$ on $M$. Then $G_{0} \cap M$ is the corresponding group for the action of $G_{0}$ and therefore $G_{0} \cap H \cong U(2)$ (see [O]). Since, by Theorem 5.4, $H$ is also isomorphic to a subgroup of $U(2)$, we must have $H=G_{0} \cap H$ and so $H \subseteq G_{0}$. It follows now from dimensional considerations that $G / H=G_{0} / H=M$ and consequently $G=G_{0}$.

\section{Main theorem.}

THEOREM 10.1. Every group $G<\mathscr{G}^{+}(M)$ is isomorphic to a subgroup of $P U(3)$ and the action of $G$ is locally complex linear.

Proof. Every reducible group embeds in $U(2)$, hence in $P U(3)$, by Theorem 5.4. For imprimitive groups this has already been shown in Theorem 6.11. Finally, the isomorphism types of primitive groups are determined by Theorems 7.5, 8.6 and Proposition 9.2. These groups also have a faithful representation in $P U(3)$ (see [B1] or Appendix 2). As noted before, the fact that all actions are locally complex linear follows from Theorem 5.4.

11. Group actions on the Chern manifold. As noted before, the classification of groups acting on $M=\mathbf{C} P^{2}$ is now completed. However, this is not the case for $M \neq \mathbf{C} P^{2}$. The problem of which groups $G \subseteq P U(3)$ can actually act on $M$ remains open.

We observe first that if $M$ admits an action of a homology then its splits off a copy of $\mathbf{C} P^{2}$, i.e., $M=\mathbf{C} P^{2} \# \Sigma$ for some homology 4-sphere $\Sigma$. Indeed, according to [F2] the fixed 2-sphere has a normal bundle, which by Proposition 2.1 and duality is equivalent to the Hopf bundle. Thus nonsplittable homology $\mathbf{C} P^{2}$ 's admit only pseudofree actions (every group element can have only isolated fixed points).

Remark. If $M$ admits a $G$-action and rank $G \geqslant 1$, there is also a restriction on the fundamental group - by $[\mathbf{P}], \pi_{1}(M)$ must be a 3-manifold group. It was also shown in $[\mathbf{P}]$ that there are infinitely many (splittable) homology $\mathbf{C} P^{2}$ 's that do not satisfy this condition. If rank $G=2$, then $M=\mathbf{C} P^{2}$ by [OR].

We turn now to the Chern manifold $\mathbf{C}$. This manifold was constructed in [F1] and it is the only closed topological manifold homotopy equivalent to $\mathbf{C} P^{2}$ but not homeomorphic to it. Kwasik showed in $[\mathbf{K}]$ that $\mathbf{C} h$ does not admit any nontrivial (locally smooth) involution. On the other hand, he also showed that every cyclic group of odd order does act on $\mathbf{C}$. A different construction of cyclic group actions on $\mathbf{C} h$ is due to Edmonds [E2]; his method works for other 4-manifolds as well. We shall refine Kwasik's argument to obtain a complete classification.

It is easy to see that $\mathbf{C} h$ is nonsplittable. For if $\mathbf{C} h \cong \mathbf{C} P^{2} \# \Sigma$, then $\pi_{1}(\Sigma)=0$ by van Kampen's theorem and hence $\Sigma \cong S^{4}$ by the topological Poincaré conjecture [F1]. This, in turn, would imply $\mathbf{C} h \cong \mathbf{C} P^{2}$. It follows that $\mathbf{C} h$ admits only pseudofree actions. Thus every $G<\mathscr{G}(\mathbf{C} h)$ is either a cyclic group of odd order or a metacyclic imprimitive group. Using the terminology from $\S 6$, the latter type is 
characterized by the property that $G=S(G)$ and $R(G)$ is cyclic. To show that $\mathbf{C} h$ supports imprimitive actions, we need a procedure to transfer actions from $\mathbf{C} P^{2}$ to $\mathrm{C} h$. This can be achieved by means of surgery theory since according to [F2], topological surgery works in dimension 4 for manifolds with finite fundamental groups. The surgery techniques yield the following result (implicitly contained in $[\mathbf{K}])$.

Proposition 11.1. Let $X$ be a compact oriented 4-manifold with boundary (possibly empty). Suppose $\pi_{1}(X)$ is a finite group of odd order. If $X$ has odd intersection form (i.e., $x \cdot x$ is odd for some $x \in H_{2}(X) /$ torsion), then there is a compact 4-manifold $Y$ and a homotopy equivalence $(Y, \partial Y) \rightarrow(X, \partial X)$, inducing a homeomorphism on the boundary, so that $X$ and $Y$ have different relative Kirby-Siebenmann invariants.

Remark. In case $\pi_{1}(X)=0$, the above statement is a part of Freedman's existence theorem $[\mathbf{F 1}]$.

Proposition 11.2. Suppose $M$ is a closed, simply connected 4-manifold with odd intersection form. Let $N$ be the unique 4-manifold that is homotopy equivalent to $M$, with different Kirby-Siebenmann invariant. If $M$ supports an action of an odd order group $G$ that is locally smooth, pseudofree, and trivial on homology, then $N$ also supports such a G-action.

REMARK. For $G$ a cyclic group, this was also shown by Edmonds in [E2].

Proof. Remove from $M$ a small open disk around each point of $\bigcup_{g \neq 1} M^{g}$ to obtain a $G$-invariant manifold $M_{1}$. Then $G$ acts freely on $M_{1}$. The action is trivial on homology and so $X=M_{1} / G$ has odd intersection form. Let $Y$ be the manifold provided by Proposition 11.1 and let $N_{1}$ be its universal cover. Define $N_{2}=N_{1} \cup$ ( $M$ - Int $M_{1}$ ) with the obvious identification along the boundary. By construction, $N_{2}$ comes equipped with an action of $G$ that has the required properties and by Freedman [F1], $N_{2} \cong N$.

Finally, note that Proposition 11.2 produces an action on $\mathbf{C} h$ when applied to a subgroup of $P U(3)$ acting pseudofreely on $\mathbf{C} P^{2}$.

Appendix 1. We assume throughout this appendix that $n>2$ is either an odd number or a power of 2 . Let $\lambda=e^{2 \pi i / m}$, where $m=n \cdot \operatorname{gcd}(2, n)$ and let $\omega=\lambda^{2}$. (In particular, $\omega$ is a primitive $n$th root of unity.)

Let $N: \mathbf{Z}[\lambda] \rightarrow \mathbf{Z}$ be the norm map.

Proposition. Let $\beta_{k}=\lambda^{k}+\lambda^{-k}$. Then

(i) $N\left(\beta_{k}\right) \neq 0 \Rightarrow N\left(\beta_{k}\right)=2^{t}$ for some $t \geqslant 0$,

(ii) $N\left(\beta_{k}\right)=0 \Leftrightarrow \beta_{k}=0 \Leftrightarrow 2 \cdot \operatorname{gcd}(k, n)=n$,

(iii) $N\left(\beta_{k}\right)=1 \Leftrightarrow n=m, \lambda^{k} \neq 1$,

(iv) $m=2 n, 2 \cdot \operatorname{gcd}(k, n)<n \Rightarrow N\left(\beta_{k}\right)=4^{\operatorname{gcd}(k, n)}$,

(v) $N\left(\beta_{k}\right) \geqslant N(2) \Leftrightarrow \beta_{k}=2 \lambda^{k}= \pm 2$,

(vi) $\beta_{k} \beta_{l}=\beta_{k+1}+\beta_{k-1}$. 
Proposition A.1. Suppose that

$$
\left(\frac{\omega^{k}+1}{\omega^{k}-1}\right)\left(\frac{\omega^{l}+1}{\omega^{l}-1}\right)=\left(\frac{\omega+1}{\omega-1}\right)^{2}
$$

for some $k, l, \operatorname{gcd}(k l, n)=1$. Then $\omega^{k}=\omega^{l}=\omega^{ \pm 1}$.

Proof. If $n=4, \omega^{k}=\omega^{l}=\omega^{ \pm 1}$ by direct verification. We shall assume that $n>4$. Due to (vi), the hypothesis can be rewritten as

$$
\frac{\beta_{k+1}+\beta_{k-1}}{\beta_{k+1}-\beta_{k-1}}=\frac{\beta_{2}+\beta_{0}}{\beta_{2}-\beta_{0}} .
$$

We cross-multiply and simplify the result:

$$
\beta_{2} \beta_{k-1}=2 \beta_{k+1} \text {. }
$$

Since $\beta_{2} \neq 0$, both sides of $(*)$ can vanish only for $\beta_{k-1}=\beta_{k+1}=0$; but then $\beta_{k} \beta_{l}=0$, which is clearly not the case. Thus, taking norms we get $N\left(\beta_{k-1}\right) \geqslant N(2)$ and consequently $\beta_{k-1}=2 \lambda^{k-1}= \pm 2$. This implies $\omega^{k-1}=1$. It follows now from $(*)$ that $\beta_{2}= \pm \beta_{2 k}$, and so $\omega^{k}=\omega^{ \pm 1}$.

Proposition A.2. Suppose that

$$
\left(\frac{\omega^{x}+1}{\omega^{x}-1}\right)\left(\frac{\omega^{y}+1}{\omega^{l}-1}\right)+2\left(\frac{\omega^{k}+1}{\omega^{k}-1}\right)\left(\frac{\omega^{l}+1}{\omega^{l}-1}\right)=1
$$

for some $x, y, \operatorname{gcd}(x y, n)=1$. If $\operatorname{gcd}(k l, n)=1$ for $n$ odd and $\operatorname{gcd}(k, n)=2$, $\operatorname{gcd}(l, n) \leqslant 2$ for $n$ even, then $\omega^{x}=\omega^{-y}$.

Proof. Again, the case $n=4$ can verified directly, so assume $n>4$. By assumption,

$$
\frac{\beta_{x+y}+\beta_{x-y}}{\beta_{x+y}-\beta_{x y}}+2 \frac{\beta_{k+1}+\beta_{k-1}}{\beta_{k+1}-\beta_{k-1}}=1 .
$$

Cross - multiply and simplify to obtain

$$
\beta_{x+y}\left(\beta_{k+1}+\beta_{k-1}\right)=2 \beta_{x-1} \beta_{k-1}
$$

or

$$
\beta_{x+1} \beta_{k} \beta_{l}=2 \beta_{x-1,} \beta_{k-1}
$$

Since $n>4$, both products in $(* *)$ are nonzero or else $\beta_{x+y}=\beta_{x-y}=0$, but this would imply $\beta_{x} \beta_{y}=0$. Thus, taking norms we see that $N\left(\beta_{x+y}\right) \geqslant N(2)$. Hence $\beta_{x+y}=2 \lambda^{x+y}= \pm 2$ and therefore $\omega^{x+y}=1$.

\section{Appendix 2. Irreducible subgroups of $P U(3)$.}

1. Imprimitive groups. Every finite imprimitive subgroup $G \subseteq P U(3)$ is conjugate to a subgroup of the form $R(G) \rtimes \varphi(G)$, where $R(G)=\Pi_{p} R(G)_{p}, R(G)_{p}=\langle g, y\rangle$ is a $p$-Sylow subgroup of $R(G)$ and $\varphi(G)=\langle u\rangle$ or $\langle u, v\rangle$ with $g, y, u, v$ defined in homogeneous coordinates as follows. 
Given a prime number $p$ and integers $n, m, r$ such that $n \geqslant m$ are powers of $p$ and $\operatorname{gcd}(r, p)=1$, let

$$
\begin{aligned}
& g\left[z_{0}: z_{1}: z_{2}\right]=\left[\omega z_{0}: \omega^{-r} z_{1}: z_{2}\right], \\
& y\left[z_{0}: z_{1}: z_{2}\right]=\left[z_{0}: \omega^{k} z_{1}: z_{2}\right], \\
& u\left[z_{0}: z_{1}: z_{2}\right]=\left[z_{2}: z_{0}: z_{1}\right], \\
& v\left[z_{0}: z_{1}: z_{2}\right]=\left[z_{1}: z_{0}: z_{2}\right],
\end{aligned}
$$

where $\omega=e^{2 \pi i / n}, k=n m$.

In addition, we also require that

(i) $n=3 m, r \equiv 1 \bmod p, r \equiv 1 \bmod m$ if $p=3, m<n$,

(ii) $r^{2}+r+1 \equiv 0 \bmod n$ if $p \neq 3, m<n$,

(iii) $\varphi(G)=\langle u\rangle$ if either $m<n$ for some $p \neq 3$ or $r \neq 1 \bmod n$ for $p=3$.

Under these conditions $R(G)$ is normalized by $\varphi(G)$ and the resulting $\varphi(G)$ action on $R(G)$ is precisely that described in Propositions 6.6-6.8.

There exists also a compact imprimitive subgroup of positive dimension. It is generated by the elements $u, v$ defined above and all transformations of the form

$$
\left[z_{0}: z_{1}: z_{2}\right] \rightarrow\left[e^{i s} z_{0}: e^{i t} z_{1}: z_{2}\right], \quad s, t \in \mathbf{R} .
$$

2. Primitive groups having normal imprimitive subgroups. Up to conjugation there are three subgroups of $P U(3)$ with this property. These are the Hessian group $H \rtimes S L(2,3)=\langle x, y, u, v, w\rangle$ and its two subgroups $H \rtimes C_{4}=\langle x, y, u\rangle, H \rtimes Q$ $=\langle x, y, u, v\rangle$ with the generators $x, y, u, v, w$ defined as follows:

$$
\begin{aligned}
& x\left[z_{0}: z_{1}: z_{2}\right]=\left[\lambda z_{0}: \lambda^{2} z_{1}: z_{2}\right], \quad \lambda=e^{2 \pi i / 3}, \\
& y\left[z_{0}: z_{1}: z_{2}\right]=\left[z_{1}: z_{2}: z_{0}\right], \\
& u\left[z_{0}: z_{1}: z_{2}\right]=\left[z_{0}+z_{1}+z_{2}: z_{0}+\lambda z_{1}+\lambda^{2} z_{2}: z_{0}+\lambda^{2} z_{1}+\lambda z_{2}\right], \\
& v\left[z_{0}: z_{1}: z_{2}\right]=\left[z_{0}+z_{1}+\lambda^{2} z_{2}: z_{0}+\lambda z_{1}+\lambda z_{2}: \lambda z_{0}+z_{1}+\lambda z_{2}\right], \\
& w\left[z_{0}: z_{1}: z_{2}\right]=\left[z_{0}: z_{1}: \lambda z_{2}\right] .
\end{aligned}
$$

Then $x^{3}=y^{3}=u^{4}=v^{4}=w^{3}=1$, and the action of $\langle u, v, w\rangle$ on $H=\langle x, y\rangle$ is given by

$$
\begin{aligned}
u x u^{-1} & =y, \quad u y u^{-1}=x^{2}, \quad v x v^{-1}=x y, \\
v y v^{-1} & =x y^{2}, \quad w x w^{-1}=x, \quad w y w^{-1}=x^{2} y .
\end{aligned}
$$

3. Simple primitive groups. (i) $\operatorname{PSL}(2,7)$.

\section{Presentation:}

$$
\left\langle u, v, w \mid u^{7}=v^{3}=w^{2}=1, u v=v u^{4}, v w=w v^{2},(w u)^{4}=1\right\rangle .
$$

Representation in $P U(3)$ :

$$
\begin{aligned}
u\left[z_{0}: z_{1}: z_{2}\right] & =\left[z_{0}: \lambda z_{1}: \lambda^{3} z_{2}\right], \quad \lambda=e^{2 \pi i / 7}, \\
v\left[z_{0}: z_{1}: z_{2}\right] & =\left[z_{1}: z_{2}: z_{0}\right], \\
w\left[z_{0}: z_{1}: z_{2}\right] & =\left[a z_{0}+b z_{1}+c z_{2}: b z_{0}+c z_{1}+a z_{2}: c z_{0}+a z_{1}+b z_{2}\right],
\end{aligned}
$$

where $a=\lambda^{3}-\lambda^{2}, b=\lambda-\lambda^{4}, c=1-\lambda^{5}$. 
(ii) $\operatorname{PSL}(2,5)$ and $\operatorname{PSL}(2,9)$.

Presentation of $P S L(2,9)$ :

$$
\begin{aligned}
\left\langle x_{1}, x_{2}, x_{3}, x_{4}\right| x_{1}^{3} & =x_{i}^{2}=1(2 \leqslant i \leqslant 4), \\
\left(x_{i} x_{i+1}\right)^{3} & \left.=1(1 \leqslant i \leqslant 3),\left(x_{i} x_{j}\right)^{2}=1(i+1<j)\right\rangle,
\end{aligned}
$$

$\operatorname{PSL}(2,5)=\left\langle x_{1}, x_{2}, x_{3}\right\rangle$.

Representation in $P U(3)$ :

$$
\begin{aligned}
& x_{1}\left[z_{0}: z_{1}: z_{2}\right]=\left[z_{2}: z_{0}: z_{1}\right], \\
& x_{2}\left[z_{0}: z_{1}: z_{2}\right]=\left[-z_{0}: z_{1}: z_{2}\right], \\
& x_{3}\left[z_{0}: z_{1}: z_{2}\right]=\left[z_{0}+a z_{1}+b z_{2}: a z_{0}+b z_{1}+z_{2}: b z_{0}+z_{1}+a z_{2}\right], \\
& x_{4}\left[z_{0}: z_{1}: z_{2}\right]=\left[z_{0}: \omega z_{2}: \omega^{2} z_{1}\right], \quad \omega=e^{2 \pi i / 3},
\end{aligned}
$$

where $a=(1+\sqrt{5}) / 2, b=(1-\sqrt{5}) / 2$.

(iii) $S O(3)$.

(iv) $P U(3)$.

\section{REFERENCES}

[AS] M. F. Atiyah and I. M. Singer, The index of elliptic operators. III, Ann. of Math. (2) 87 (1968), $546-604$.

[B1] H. F. Blichfeldt, Finite collineation groups, Univ. of Chicago Press, Chicago, 1917.

[B] G. E. Bredon, Introduction to compact transformation groups, Academic Press, New York, 1972.

[Br] K. S. Brown, Cohomologv of groups, Springer-Verlag. New York, 1982.

[CS] S. E. Cappel and J. L. Shaneson, Nonlinear similarity and linear similarity are the same in dimensions less than 6, preprint.

[CF] P. E. Conner and E. E. Floyd, Maps of odd period, Ann. of Math. (2) 84 (1966), 132-156.

[CM] H. S. M. Coxeter and W. O. J. Moser, Generators and relations for discrete groups, 4th ed., Springer-Verlag, Berlin and New York, 1980.

[D] K. H. Dovermann, Rigid cyclic group actions on cohomologv complex projective spaces, preprint.

[E1] A. L. Edmonds, Transformation groups and low-dimensional manifolds, Contemporary Math., vol. 36, Amer. Math. Soc., Providence, R. I., 1985, pp. 339-366.

[E2] Construction of group actions on four-manifolds, Trans. Amer. Math. Soc. 299 (1987), $155-170$.

[F1] M. H. Freedman, The topology of four-dimensional manifolds, J. Differential Geometry 17 (1982), $357-453$.

[F2] The disk theorem for four-dimensional manifolds, Proc. Internat. Congr. Math.. Warsaw, 1983, pp. 647-663.

[G] D. Corenstein. The classification of finite simple groups, Plenum, New York, 1983.

[HY] A. Hattori and T. Yoshida, Lifting compact group actions in fiber bundles, Japan. J. Math. 2 (1976), 13-25

[H] B. Huppert, Endliche Gruppen. I, Springer-Verlag, Berlin and New York, 1967.

[K] S. Kwasik, On the symmetries of the fake C $P^{2}$, Math. Ann. 274 (1986), 385-389.

[O] H. Oh. Compact connected Lie groups acting on simply connected 4-manifolds, Pacific J. Math. 109 (1983), 425-436.

[OR] P. Orlik and F. Raymond, Actions of the torus on 4-manifolds. I, Trans. Amer. Math. Soc. 152 (1979). 531-559.

[P] S. P. Plotnick. Circle actions and fundamental groups for homology 4-spheres, Trans. Amer. Math. Soc. 273 (1982), 393-4()4. 
[R] J. J. Rotman, An introduction to the theory of groups, 3rd ed., Allyn and Bacon, Boston, Mass., 1984. [SW] O. Schreier and B. L. van der Waerden, Die Automorphismen der Projektiven Gruppen, Abh. Math. Sem. Univ. Hamburg 6 (1928), 303-322.

[S] R. Schultz, On the topological classification of linear representations, Topology 16 (1977), 263-270.

[W] C. T. C. Wall, Surgery on compact manifolds, Academic Press, London, 1970.

[Wo] J. A. Wolf, Spaces of constant curvature, 4th ed., Publish or Perish, 1977.

Department of Mathematics, Indiana University, Bloomington, Indiana 47405

Current address: Department of Mathematics, Yale University, New Haven, Connecticut 06520 